\title{
Ultrasound as an aid for reduction of paediatric forearm fractures
}

\author{
Christopher Ern-Yoong Wong • Angelina Su-Yin Ang • \\ Kee-Chong Ng
}

Received: 21 February 2008 / Accepted: 23 September 2008 / Published online: 19 November 2008

(C) Springer-Verlag London Ltd 2008

\begin{abstract}
Background Displaced distal forearm fractures are frequently reduced in emergency departments. Not infrequently, some are not done adequately and require the tedious process of repeating the procedure, with repeated X-rays and radiation exposure, and inconvenience to patient and staff. The use of ultrasound (US) in its expanding role in the practice of emergency medicine has been proposed to visualise bone positioning.

Aim Our department embarked on this proof of concept study to assess the usefulness of this tool.

Method By way of convenience sampling, we looked at whether our US interpretation correlated with the corresponding X-ray findings, pre and post manipulation of suitable fractures.

Results Out of 42 patients recruited over a 1-year period, we were successful in 38 (90\%). Four were "unsuccessful" $(10 \%)$ due to technique rather than equipment or patient factors.
\end{abstract}

C. E.-Y. Wong • A. S.-Y. Ang · K.-C. Ng

Department of Emergency Medicine,

KK Women and Children's Hospital,

Singapore, Singapore

A. S.-Y. Ang

e-mail: Angelina.Ang.SY@kkh.com.sg

K.-C. $\mathrm{Ng}$

e-mail: Ng.Kee.Chong@kkh.com.sg

Present address:

C. E.-Y. Wong $(\bowtie)$

Emergency Department, Nepean Hospital,

Penrith, NSW, Australia

e-mail: drchristopherwong@yahoo.com.au
Conclusion Whilst before we were blind prior to a postreduction X-ray, this "new" additional role of the ultrasound (very accessible, cost effective and safe) can now aid us in our decision making, thereby enhancing the work flow of this group of patients through the department.

Keywords Ultrasound · Forearm fracture - Procedural sedation and analgesia . Work flow · Conscious sedation

\section{Introduction}

The Emergency Department of the KK Women's and Children's Hospital in Singapore sees an average of 300 to 350 children per day. Of these, $15 \%$ to $20 \%$ are for trauma. Displaced distal forearm fractures contribute significantly to these trauma figures. These particular fracture types represent a pathology that can be readily corrected within the department, negating the need for an admission [1].

Such fractures are typically manipulated and reduced $(M \& R)$ in the procedure room, employing the use of either Bier's block (via regional anaesthesia) or through procedural sedation and analgesia (formerly known as conscious sedation), where ketamine is the most common drug used $[2,3]$. After the manipulation, the clinician will attempt to determine if adequate reduction has been achieved by palpating for the alignment of the bony edges of the distal end of the ulna and radius, and the relative positions of the radial and ulnar styloid [4] (see Fig. 1). The patient is then sent for a post-reduction confirmation check X-ray for satisfactory alignment. Unfortunately, there are occasions where palpating for these landmarks becomes difficult, such as in the presence of surrounding soft tissue swelling, and the inadequate alignment is only discerned from the check X-ray. 
Fig. 1 X-ray of the distal radius; inter-styloid distances shown by white arrow

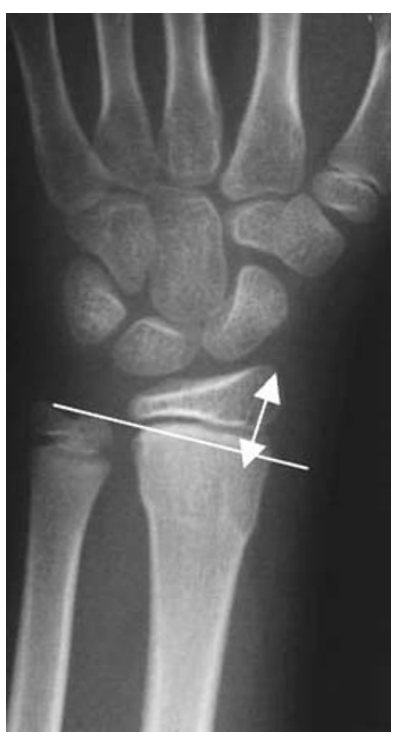

The patient will then need another M\&R to achieve adequate fracture alignment. This need for repeat X-rays takes time, and there have been frequent occasions where the sedation needs to be augmented to maintain adequate sedation and analgesia for the patients for such repeat M\&Rs.

The idea of using ultrasound as an adjunct to visualize the end product, whilst still in the procedure room, has been proposed in various case studies [5]. We embarked on this proof of concept study to assess the potential usefulness of this tool.

\section{Patients and method}

The study was conducted between September 2001 and August 2002 at the Emergency Department of the KK Women's and Children's Hospital in Singapore, an 800-bed referral hospital that sees patients aged 0 to 16 years. The patients were considered for the study after diagnosis by clinical examination and screening X-rays. The inclusion criteria were distal forearm fractures that were closed (simple), not intra-articular, non-comminuted and considered by the attending emergency specialist to be amenable to manipulation and reduction within the department. Fractures that were open or part of a patient's multiple injuries were excluded. Also excluded were those that needed operative intervention for stability, such as for the Monteggia and Galeazzi varieties of fracture.

Recruitment was by convenience sampling. The principal investigators were present to perform these ultrasounds. The investigators involved were attending ED physicians who had undergone accredited training on basic ultrasound use and were by then proficient in the use of US in FAST (Focused Assessment with Sonography in Trauma).
For data collection, the participating clinician completed a standardised form. The following parameters were collected (Table 1).

With the initial X-rays showing the fracture as a guide, a pre-manipulation US was done. We used the $7.5-\mathrm{MHz}$ linear transducer from a Toshiba Justvision 2000 DualProbe Ultrasound System. The ultrasound transducer is placed lengthwise over the fracture site along two planes, specifically the dorsal and lateral surfaces. Ultrasound coupling gel is placed between the skin and transducer to enhance the image (Fig. 2a). The dorsal cortex of the bone is clearly seen as a bright white line. The fracture, including its displacement, is readily visualized (Fig. 2b). The above is further illustrated in Fig. 2c to $\mathrm{f}$.

After the initial M\&R, confirmation of adequate fracture reduction whilst in the procedure room was supplemented with the ultrasound by looking for alignment of the bony cortex in the two planes described (Fig. 3a,b). If the initial reduction did not appear adequate, the fracture was remanipulated, and the alignment was re-confirmed with US. This process was repeated until reduction was considered satisfactory or until clinical judgment dictated that further attempts at closed reduction were unlikely to bring about any further improvement.

The fracture is immobilized with a suitable splint, and post-reduction X-rays were subsequently done to verify that reduction was satisfactory (Fig. 4a,b).

For record-keeping purposes, both hard and soft copies of the representative US views were taken.

The use of X-rays before and after manipulation follows the standard practice for fracture management. US, also done before and after manipulation, was considered corresponding with the X-rays when the interpretations of the two imaging modalities coincided.

Table 1 Data parameters collected by clinicians

Patient parameters

Gender

Age

Date of attendance

Fracture type: isolated radius/ulna/combination of both

Fracture displacement:

Degree of angulation

Contact surface (i.e., estimated proportion of end-to-end contact of

fracture fragments, or if fragments overlapped)

Age of fracture: $<24 \mathrm{~h}$ or $>24 \mathrm{~h}$

Any associated tissue swelling

Sedation type: ketamine/Bier's block/nitrous oxide

US exam results pre and post manipulation and if they corresponded with radiological exams 
Fig. 2 a, b, c Angulated midshaft fracture of the radius and ulna (lateral view); arrows indicate the approximate position of the US probe on the radius. d AP view of fracture shown in Fig. 2c; arrow indicates the approximate position of the US probe on the radius. e Corresponding US view of fracture shown in Fig. 2c; arrows indicate cortex of bone. $\mathbf{f}$ Corresponding US view of fracture shown in

Fig. 2d; arrows indicate cortex of bone

Fig. 3 a Cortex of bone indicated by arrows. b Cortex of bone indicated by arrows
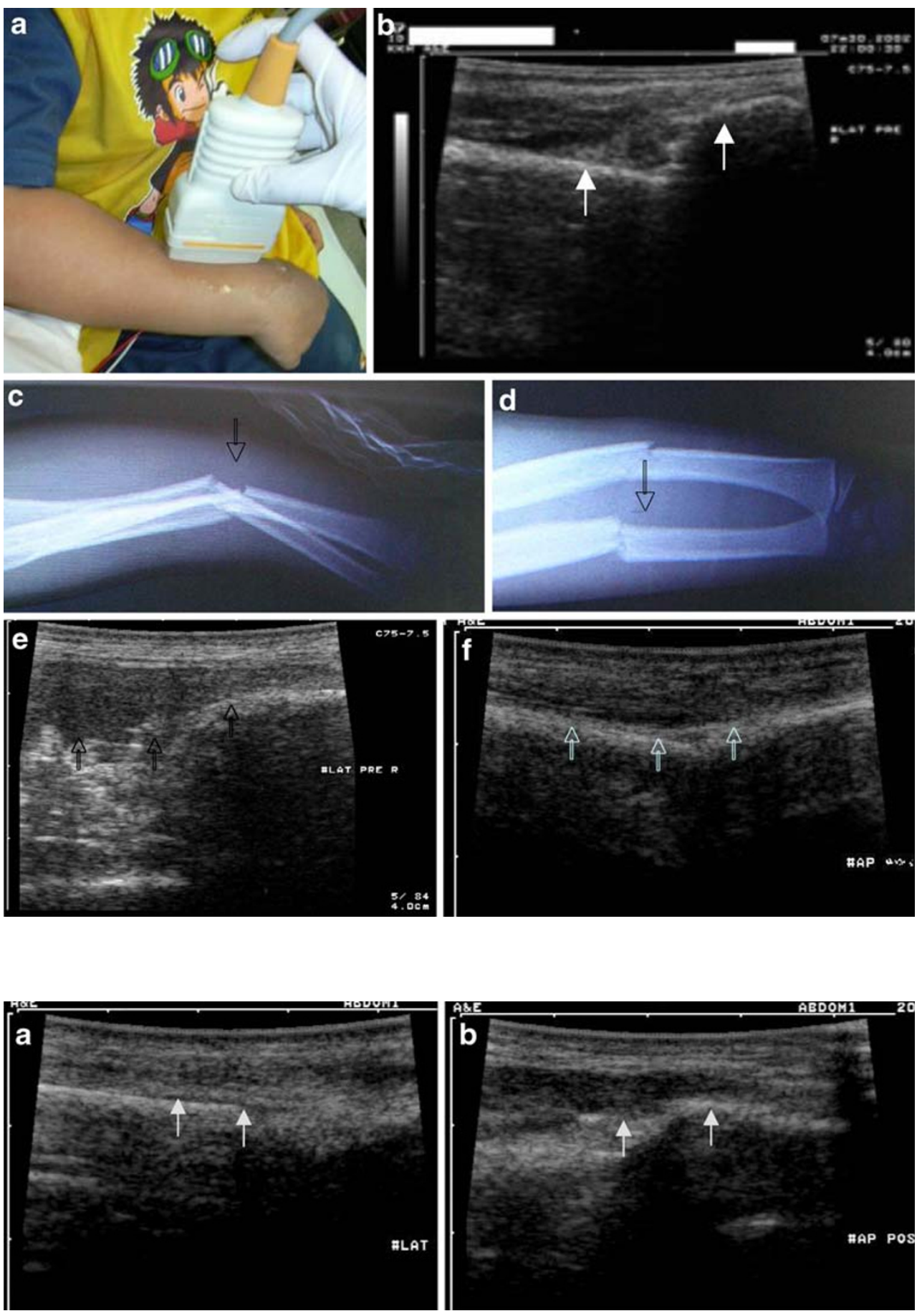

Fig. 4 a Lateral view of reduced fracture; arrow indicates approximate position of US probe. b AP view of reduced fracture; arrow indicates approximate position of US probe
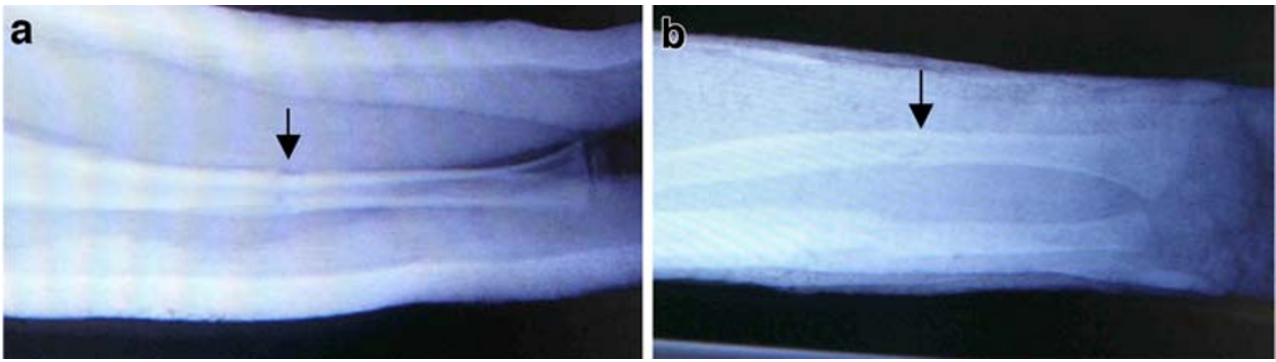
Table 2 Description of sedation

\begin{tabular}{ll}
\hline Sedation type & Number (\%) \\
\hline Ketamine & $30(71 \%)$ \\
Bier's block & $11(26 \%)$ \\
Nitrous oxide & $2(3 \%)$ \\
\hline
\end{tabular}

One patient received a Bier's block that progressed to ketamine sedation

\section{Results and observations}

During the study period, 42 cases were evaluated (Tables 2, 3 and 4). The ages ranged from 1 year to 14 .

Listed below are details (comments in italics) of the four cases (Table 5) where US interpretation did not correspond with X-ray diagnosis, which incidentally occurred early in the study (and was also not isolated to any particular author):

1. 4 year old, presented within 24 hours; swelling present; ketamine used:

Distal radial fracture with 30-degree angulation, not corrected despite manipulation, was not obvious on ultrasound.

2. 12 year old, presented within 24 hours; swelling present; Bier's block and repeated attempts with ketamine:

Distal radial and ulnar fracture with bayoneted (overlapping) displaced fragment was not reduced despite manipulation; unreduced fragments were not seen on ultrasound after manipulation.

3. 9 year old, no swelling; Bier's block:

Displaced distal radial fragment that was overlapping laterally.

4. 14 year old, swelling present; Bier's block:

There was a bayoneted (overlapping), distal ulnar fragment.

Table 3 Description of fractures

\begin{tabular}{|c|c|}
\hline & Number $(\%)$ \\
\hline \multicolumn{2}{|l|}{ Fracture type } \\
\hline Radius only & $16(38 \%)$ \\
\hline Radius and ulna & $26(62 \%)$ \\
\hline Ulna only & 0 \\
\hline \multicolumn{2}{|l|}{ Fracture swelling } \\
\hline Present & $19(45 \%)$ \\
\hline Absent & $23(55 \%)$ \\
\hline \multicolumn{2}{|l|}{ Fracture displacement } \\
\hline Angulated (requiring manipulation) & $19(45 \%)$ \\
\hline Overlapping (bayoneted) & $23(55 \%)$ \\
\hline
\end{tabular}

Table 4 US correlation with radiological exam

\begin{tabular}{lll}
\hline & Yes & No \\
\hline $\begin{array}{l}\text { Did the US exam correspond with radiological } \\
\text { exam? }\end{array}$ & & \\
Pre-manipulation & 42 & 0 \\
Post-manipulation & $(100 \%)$ & \\
\hline
\end{tabular}

The authors acknowledge the bias posed by performing the premanipulation US after viewing the initial X-rays.

Overlapping fragments were initially difficult to differentiate on ultrasound as the fragments gave the impression of alignment when viewed in a perpendicular plane. However, with progress of the study, the authors learnt to recognize this and avoided the error.

\section{Limitations of the study}

This study design was put together amongst the authors over a relatively short period of time and was set up to provide an indication of the potential use of ultrasound in the management of particular fracture patterns within the paediatric population, and by extension potentially the adult population. The recruitment of patients by convenience sampling resulted in a small power for the study. The authors have acknowledged these limitations. A better designed protocol, including a solid statistical analysis plan, has been proposed to be included in a future study to provide confirmatory evidence on the use of ultrasound in such a clinical setting.

\section{Discussion}

As described above, clinically assessing the adequacy of a fracture reduction may at times be challenging, in particular in the presence of associated soft tissue oedema over the fracture site or, alternatively, if the patient is obese. Some studies have placed the success rate at approximately $80 \%$ regardless of patient selection [6]. Post-reduction X-rays are part of standard practice to confirm the adequacy of the reduction.

Table 5 US correlation with X-rays

\begin{tabular}{|c|c|}
\hline US correlating & $38(90 \%)$ \\
\hline Reduced & 36 \\
\hline Not reduced & $\begin{array}{l}2 \text { (and therefore admitted for reduction } \\
\text { under GA) }\end{array}$ \\
\hline $\begin{array}{l}\text { US not correlating with } \\
\text { X-rays }\end{array}$ & $4(10 \%)$ (see details below) \\
\hline
\end{tabular}


In the event that post-reduction X-rays identify inadequate reduction, often another attempt at reducing the fracture occurs. To repeat the reduction attempt, the splint placed after the initial attempt is removed to allow further manipulation of the fracture and re-splinted upon completion of the procedure, with additional confirmatory X-rays performed. Throughout this process, additional sedation may be required. These extra steps to achieve satisfactory fracture reduction consequently incur additional hidden costs in terms of time and resources to an already busy emergency department and also create unnecessary stress for the patients and their caregivers [7].

The use of portable fluoroscopy in the ED has been a consideration for use in the context of support for management of fracture reduction. This process proposes the extension of the use of fluoroscopy beyond the operating theatres. The proposition of using fluoroscopy in this context gives the advantage of imaging the manipulation/reduction process in real time [8]. Attending disadvantages of using fluoroscopy to support fracture reduction are: the exposure to radiation of both the patient and staff [9], and the need for additional training. Acquisition of the appropriate licence, raising funds for new equipment and storage space are perhaps other minor issues against it.

The role of ultrasound in the emergency department has expanded exponentially over the past decade, with many departments having their own dedicated machine [10]. It is a safe and non-invasive modality. Its portability in the emergency department allows for faster decision making. A prominent example is its role in FAST (focused assessment with sonography in trauma) where its use in trauma patients has become established practice.

An ultrasound probe in the hands of a physician is rapidly becoming the "stethoscope" of the 21 st century. This study demonstrates an additional use of the US as a visual aid to fracture reduction. US provides a means to assess by the bedside the adequacy of the reduction by placing a US probe over the fracture site, not only giving on the spot reassur- ances to the clinician, but also, we hope, significantly reducing the number of repeated procedures and trips to the medical imaging department for repeat X-rays. Thereby, patient flow is improved, and, more importantly, patient satisfaction and enhancement of their care.

Conflicts of interest None.

\section{References}

1. Jones K, Weiner DS (1999) The management of forearm fractures in children: a plea for conservatism. J Pediatr Orthop 19:811-815

2. Ng KC, Ang SY (2002) Sedation with ketamine for paediatric procedures in the emergency department: a review of 500 cases. Singapore Med J 43:300-304

3. McCarty EC, Mencio GA, Walker LA, Green NE (2000) Ketamine sedation for the reduction of children's fractures in the emergency department. J Bone Joint Surg Am 82-A:912-918

4. Dée W, Klein W, Rieger H (2000) Reduction techniques in distal radius fractures. Injury 31(Suppl 1):48-55

5. Durston W, Swartzentruber R (2000) Ultrasound guided reduction of pediatric forearm fractures in the ED. Am J Emerg Med 18 (1):72-77

6. Proctor MT, Moore DJ, Paterson JM (1993) Redisplacement after manipulation of distal radial fractures in children. J Bone Joint Surg Br 75:453-454

7. Shobha Malviya et al (2000) Prolonged recovery and delayed side effects of sedation for diagnostic imaging studies in children. Pediatrics 105(3):e42, March

8. Lee SMK, Orlinsky M, Chan LS (1994) Safety and effectiveness of portable fluoroscopy in the emergency department for the management of distal extremity fractures. Ann Emerg Med 24:725-730

9. Brenner DJ, Doll R, Goodhead DT et al (2003) Cancer risks attributable to low doses of ionizing radiation: assessing what we really know. Proc Natl Acad Sci USA 100(24):1376113766

10. Richard Lanoix MD et al (2000) A preliminary evaluation of emergency ultrasound in the setting of an emergency medicine training program. Am J Emerg Med 18(1):41-45, January 